\title{
COMPARISON OF SUPRAGLOTTIC AIRWAY DEVICE I-GEL WITH CLASSIC LARYNGEAL MASK AIRWAY AND PROSEAL LARYNGEAL MASK AIRWAY FOR SHORT SURGICAL PROCEDURES
}

\author{
Monika Gandhi' ${ }^{1}$ K. K. Arora², Akansha Sharma ${ }^{3}$ \\ ${ }_{1}^{1}$ Associate Professor, Department of Anaesthesiology, MGM Medical College, Indore, Madhya Pradesh. \\ 2Professor and HOD, Department of Anaesthesiology, MGM Medical College, Indore, Madhya Pradesh. \\ ${ }^{3}$ Ex-Resident, Department of Anaesthesiology, MGM Medical College, Indore, Madhya Pradesh.
}

\section{ABSTRACT}

\section{BACKGROUND}

Supraglottic airways are airway devices for providing ventilation, oxygenation and anaesthetic gases to the patients. The SGA has revolutionised airway management because of its ease of insertion, hands-free operation, reliable airway and haemodynamic stability. The present study is conducted to compare the newer non-inflatable cuffed I-Gel with Classic LMA and Proseal LMA.

Aims and Objectives- To compare the Supraglottic Airway device I-Gel with Classic LMA and Proseal LMA for airway management in anaesthetised spontaneously ventilating patients.

\section{MATERIALS AND METHODS}

120 patients of ASA I and II scheduled for routine short surgical procedure (lasting upto 1 hour) were randomly selected. Patients were divided into three Groups I, C, P. (40 in each group). All patients pre-medicated with Inj. Glycopyrrolate $0.004 \mathrm{mg} / \mathrm{kg}$, Inj. Midazolam $0.02 \mathrm{mg} / \mathrm{kg}$, Inj. Ranitidine $1 \mathrm{mg} / \mathrm{kg}$ and Inj. Metoclopramide $0.1 \mathrm{mg} / \mathrm{kg}$. Patients were induced with Inj. fentanyl 2 $\mathrm{mcg} / \mathrm{kg}$ and Inj. propofol $2 \mathrm{mg} / \mathrm{kg}$. A proper size Supraglottic Airway Classic LMA (Group C), Proseal LMA (Group P) and I-Gel (Group I) was inserted. Maintenance was done with Oxygen, Nitrous Oxide and Isoflurane. Time of insertion of device, number of attempts for insertion, any complications, haemodynamic parameters ( $\mathrm{HR}, \mathrm{MAP}, \mathrm{SpO}_{2}, \mathrm{ETCO}_{2}$ ) and airway leak pressure were noted. After completion of the procedure, the Supraglottic Airway was removed.

\section{RESULTS}

Meantime for insertion in I Group (12.13 \pm 1.48$)$ sec is less compared to Group C $(16.25 \pm 1.032)$ and Group P $(19.48 \pm 1.710)$. The difference was statistically significant with $P$ value $<0.001$. The mean numbers of attempt in three groups are similar. The success rate of insertion in the first attempt is not statistically significant. HR and MAP did not show any difference in the three groups. Group I and Group P have significant higher value of leak pressure compared to Group C and statistically significant. Higher incidence of post-operative sore throat was observed in Group C.

\section{CONCLUSION}

I-Gel, the new supraglottic device is easier to insert with short time and high success rate of insertion in first attempt. I-Gel has a slightly higher leak pressure compared to classic LMA with no incidence of post-operative sore throat. I-Gel is the better alternative to other supraglottic device.

\section{KEYWORDS}

I-Gel, LMA, Proseal LMA, Supraglottic Airway Device, Short Surgical Procedures.

HOW TO CITE THIS ARTICLE: Gandhi M, Arora KK, Sharma A. Comparison of supraglottic airway device I-Gel with classic laryngeal mask airway and proseal laryngeal mask airway for short surgical procedures. J. Evolution Med. Dent. Sci. 2018;7(18): 2222-2226, DOI: $10.14260 / \mathrm{jemds} / 2018 / 500$

\section{BACKGROUND}

For decades, endotracheal intubation or bag-and-mask ventilation were the mainstays of airway management. The introduction of first supraglottic airway device, laryngeal mask airway (LMA) by Brain in 1983 revolutionised the method of airway management. The device is better than face mask, as it provides a reliable ventilation and hands-free operation. The ease and speed of placement, less sympathetic stimulation and adverse effects and secure ventilation makes it an alternative to ET.(1)

'Financial or Other Competing Interest': None.

Submission 04-04-2018, Peer Review 15-04-2018,

Acceptance 18-04-2018, Published 30-04-2018.

Corresponding Author:

Dr. Monika Gandhi,

\#91/3, Vallabh Nagar,

Indore-452003,

Madhya Pradesh.

E-mail: drmonikagandhi2911@gmail.com

DOI: $10.14260 /$ jemds $/ 2018 / 500$

\section{(c) $(1) \ominus$}

There is a tremendous increase in number of day care surgeries. For this, always there has been a search and need for ways which can make outpatient anaesthesia safe and efficient. Agents and methods which provide faster induction and recovery, less adverse effects, less hospital stay are preferable and in demand. It is found that SGA are well suited for day care anaesthesia than ET.

Insertion of SGA is especially useful in patients with coronary artery disease as compared to ET, as it causes less sympathetic stimulation, thus reducing the risk of adverse cardiovascular changes. SGAs are found to be suitable during light plane of anaesthesia as compared to ET.(2)

The incidence of postoperative sore throat is also significantly less in patients receiving the LMA.(3,4) Another advantage is that an SGA typically does not require neuromuscular blockade, thereby avoiding any associated morbidity and side effects of the medication or its antagonists.

To be clinically efficient, SGA must provide a proper oropharyngeal seal, provide a low resistance to gas flows, 
suitable during both spontaneous and positive pressure ventilation, protection of airway from aspiration of secretions and gastric contents, minimal adverse effects. There are many SGAs available, but suitability and success depends on its efficiency and acceptance. $(5,6)$

Compared with ETs, SGAs only partially protect against aspiration of gastric contents. This limitation precludes their use in patients with a full stomach or other risk factors for aspiration. Delivery of positive pressure ventilation is limited by the SGA's airway leak pressure, which for many lies between 20 and $25 \mathrm{~cm} \mathrm{H}_{2} \mathrm{O} .(7,8)$

The enormous success of the LMA has been followed by the proliferation of other supraglottic airway devices, each claiming advantages over devices already in use.(9) The newer SGAs- LMA supreme and I-Gel awaits clinical studies to prove their efficacy and safety in patients.

The LMA classic was originally developed for use during routine general anaesthesia with spontaneous ventilation. The device can also be used with positive pressure ventilation at peak airway pressures not exceeding 20 to 25 $\mathrm{cm} \mathrm{H}_{2} \mathrm{O}$ or with pressure support ventilation. Although, designed for elective airway management, it has been used successfully as an airway rescue device in emergencies including resuscitation. The LMA classic remains the most widely used SGA.

The ProSeal LMA(10) was introduced in 2000 as a modification of LMA classic with a better airway seal and an added oesophageal drain tube to separate the gastric and respiratory tracts. These features improve the safety and efficacy of positive pressure ventilation, provide a means of gastric suctioning, reduce the risk of regurgitation and aspiration of gastric contents and help confirm correct mask position.

I-Gel(11) is the latest addition to the supraglottic airway devices arsenal. It is designed to create a non-inflatable, anatomic seal of the pharyngeal, laryngeal and perilaryngeal structures and avoid compression trauma.

In this study, we aim to compare the ease of insertion of IGel with Classic and Proseal LMA in short surgical procedure.

\section{MATERIALS AND METHODS}

The study was carried out in the Department of Anaesthesiology, MGM Medical College, Indore. It was a prospective, randomised, controlled, comparative study done over a definite period of time over 120 patients posted for short surgical procedures in routine theatre list. The sample size of 120 patients was taken for convenience. Exclusion criteria included patients with decreased lung compliance, hypertensive and diabetic patients, patients with increased risk of aspirations, difficult intubation, coagulopathy and obese patients and with deranged renal function. After taking permission from the Institutional Ethics Committee, 120 patients of ASA grade 1 and 2 who were posted for short surgical procedures (lasting upto 1 hour) were selected.

After taking proper written informed consent from the patient, randomisation was done by computer generated numbers.

Patients were divided into three groups I, C and P ( $n=40$ in each group). The patients were divided into 3 groups randomly through computer generated numbers.

A thorough pre-anaesthetic check-up was done prior to the planned procedure. The patients were asked to undergo a preoperative fasting of 6 hours. Patients were pre-medicated with Inj. Glycopyrrolate $0.004 \mathrm{mg} / \mathrm{kg}$ and Inj. Midazolam 0.02 $\mathrm{mg} / \mathrm{kg}$, Inj. Ranitidine $1 \mathrm{mg} / \mathrm{kg}$ and Inj. Metoclopramide 0.1 $\mathrm{mg} / \mathrm{kg}$ half an hour prior to induction. In the operation theatre, the recording of routine vital parameters was taken.

Patients received Inj. fentanyl in the dosage of $2 \mathrm{mcg} / \mathrm{kg}$. After pre-oxygenation for 5 mins. anaesthesia was induced with Inj. propofol titrated to loss of verbal contact, loss of eye lash reflex and relaxation of jaw. After confirming the possibility of bag and mask ventilation the proper size of supraglottic airway according to weight of the patient, Classic LMA (Group C), LMA Proseal (Group P) or I-Gel (Group I) was inserted. The LMA was inflated with $20 \mathrm{~mL}$ air. Then the device was connected to the breathing circuit. Confirmation of adequate placement was done by seeing the square wave pattern on $\mathrm{ETCO}_{2}$ and observing for adequate chest rise on squeezing the reservoir bag.

Time of insertion (The time from grasp of device till the confirmation of adequate placement) is recorded.

The number of attempts for insertion was also recorded. The device was considered to be successfully placed in upto 3 attempts. More than three attempts was considered as failure.

Any complication while inserting the device like coughing, gaging or vomiting was looked for and was noted.

Haemodynamic parameters (Heart rate, Mean arterial pressure, $\mathrm{SpO}_{2}$ and End tidal $\mathrm{CO} 2$ ) of the patient were recorded at 1, 3, 5 and 10 minutes after insertion.

The airway leak pressure was assessed by closing the expiratory valve of the circle system at a fixed gas flow of 3 litres/ min, noting the airway pressure (maximum allowed was $40 \mathrm{~cm} \mathrm{H}_{2} \mathrm{O}$ ). At this time, gas leaks at the sealing pressure were evaluated by auscultation using a stethoscope placed at the patient's mouth.

\section{Statistics}

Continuous variables are described as mean \pm SD and the difference between the three groups were analysed using one-way ANOVA followed by post-hoc Tukey's test. Categorised variables were described as frequency and analysed by Pearson's Chi-Square test. $\mathrm{P}$ value $<0.05$ was considered significant. SPSS software was used for the analysis of data.

\section{RESULTS}

\begin{tabular}{|c|c|c|c|}
\hline & I Group & C Group & P Group \\
\hline Mean age (years) & 33.925 & 36.55 & 35.18 \\
\hline \multicolumn{3}{|c|}{ Average Age in Three Groups } \\
\hline
\end{tabular}

The three groups are comparable in relation to age showing no significant difference in the mean age among the three groups.

\section{Weight}

Average weight in the three groups was 59.975, 58.9 and 58.875 kgs in I, C and P groups respectively. There was no significant difference.

\begin{tabular}{|c|c|c|c|}
\hline & I Group & C Group & P Group \\
\hline Mean Time (Sec) & $12.13 \pm 1.48$ & $16.25 \pm 1.032$ & $19.48 \pm 1.710$ \\
\hline \multicolumn{4}{|c|}{ Time of Insertion }
\end{tabular}


Mean time required for the insertion in I Group (12.13 seconds) is less compared to C Group (16.25 seconds) and $\mathrm{P}$ Group (19.48 seconds). The difference among the groups is statistically significant with a $P$ value of $<0.001$.

\begin{tabular}{|c|c|c|c|}
\hline Groups & I Group & C Group & P Group \\
\hline Mean No. of attempts & $1.05 \pm 0.22$ & $1.10 \pm 0.379$ & $1.20 \pm 0.516$ \\
\hline \multicolumn{3}{|c|}{ No. of Attempts of Insertion } \\
\hline
\end{tabular}

The mean no. of attempts in the three groups are similar. There is no statistically significant difference among the three groups ( $\mathrm{P}$ value of 1.000).

The success rate of insertion in the first attempt does not show any statistically significant difference $(P>0.05)$ among the three groups.

\section{Heart Rate (Beats Per Minute)}

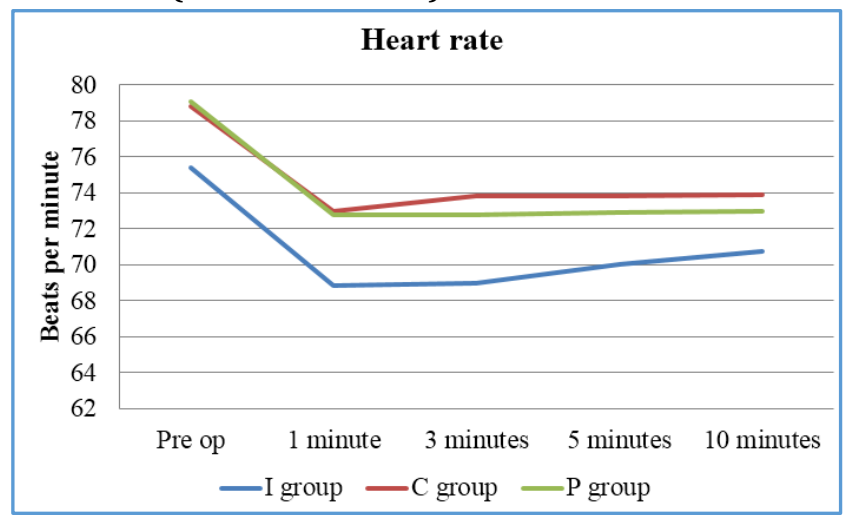

The preoperative heart rate variations in three groups are comparable. The mean heart rate intraoperatively is less compared to preop values in all the three groups. The comparison of three groups at different time intervals $(1 \mathrm{~min}$, 3 mins, 5 mins) shows that the heart rate of Group (I) is significantly low as compared to other groups. It is not significant at 10 mins.

Mean arterial pressure variation in the three groups does not show any significant difference. The comparison of three groups at all intraoperative time intervals show that MAP of Group (P) is significantly lower than other groups.

\section{Mean Arterial Pressure}

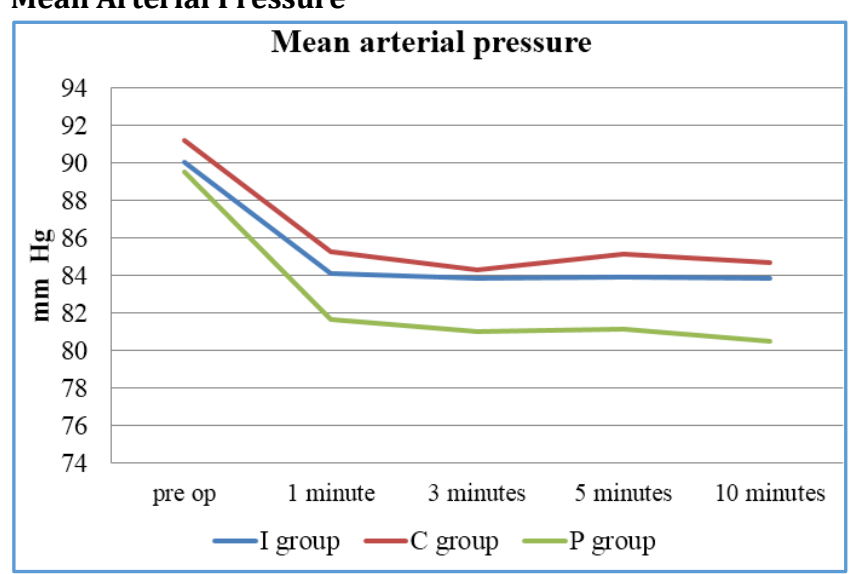

I and $\mathrm{P}$ groups have a significantly higher values $(26.23$ and $27.75 \mathrm{~cm}$ of $\mathrm{H}_{2} \mathrm{O}$ ) of leak pressure compared to $\mathrm{C}$ group where the leak pressure is $20.58 \mathrm{~cm}$ of $\mathrm{H}_{2} \mathrm{O}$. This difference among the groups is statistically significant giving a $\mathrm{P}$ value of 0.000 .

\begin{tabular}{|c|c|c|c|}
\hline & Group I & Group C & Group P \\
\hline $\begin{array}{c}\text { No. of cases of postop sore } \\
\text { throat }\end{array}$ & $0 / 40$ & $7 / 40$ & $2 / 40$ \\
\hline Percentage & 0 & $17.5 \%$ & $5 \%$ \\
\hline \multicolumn{2}{|c|}{ Postoperative Sore Throat } \\
\hline
\end{tabular}

There is a significantly higher incidence $(17.5 \%)$ of postoperative sore throat in Group C compared to both I and $\mathrm{P}$ groups where the incidence is 0 and $5 \%$ respectively.

The only complication which was seen after removing was blood staining of the device- Group I (1/40), Group C $(3 / 40)$ and Group $P(5 / 40)$. The difference in the three groups is not significant.

\section{DISCUSSION}

In our study, 120 patients of the age group 18 to $60 \mathrm{yrs}$. of ASA grade 1 and 2 were included. All these patients were comparable in regard to their demographic profile- age, sex and weight distribution.

The groups were comparable as far as type and duration of surgery were concerned.

The mean time of insertion in Group I was $12.13 \pm 1.485$ seconds, in Group C was $16.25 \pm 1.032$ seconds and in Group $P$ it was $19.48 \pm 1.710$ seconds. The difference in time of insertion is gross and is statistically significant giving a $P$ value of $<0.001(0.000)$. Time required for insertion of I-Gel was significantly less.

Our study results go in hand with the study done by Hossam et al,(12) where the mean duration of insertion attempts was $15.6 \pm 4.9$ seconds in the I-Gel group, while it was $26.2 \pm 17.7$ seconds in the LMA group. The difference between both groups regarding duration of insertion attempts was statistically significant $(\mathrm{P}=0.0023)$.

The mean no. of attempts of insertion in the Group I was $1.05 \pm 0.221$. The mean no. of attempts of insertion in Group $C$ was $1.10 \pm 0.379$. The mean no. of attempts of insertion in Group $\mathrm{P}$ was $1.20 \pm 0.516$. The success rate of insertion in first attempt in Groups I, C and P were 95\%, 92.5\% and 90\% respectively and there was no statistically significant difference.

Richez et al(11) observed that there was $97 \%$ success rate of insertion of I-Gel in the first attempt. Similar findings were also observed by Acott(13) in patients during general anaesthesia. Gatward et al(14) also found maximum success rate of insertion in first attempt in non-paralysed patients. These findings were in accordance with the results of our study, which showed that in majority of patients the insertion of I-Gel was successful in first attempt.

A study done by Hossam et al(12) for the comparison of IGel and Classic LMA in anaesthetised spontaneously ventilating patients showed that insertion and ventilation was possible at the first attempt in $90 \%$ of patients in the IGel group and in $80 \%$ in LMA group. The number of insertion attempts was statistically insignificant between both the study groups $(\mathrm{P}>0.05)$.

Our result is also supported by studies done by $\mathrm{L}$. Gasteiger et al.(15) In a non-crossover study they had found that first attempt and overall insertion success were similar 
(LMA ProSeal, 75/76 (99\%) and 76/76 (100\%); I-Gel 73/75 (97\%) and 75 (100\%), respectively).

We did not find a significant difference in the haemodynamics of the patients in the three groups. There was a significant decrease in the Heart rate and Mean arterial pressure after insertion of device from the preoperative values. That might be just because of anaesthetic depth attained by the patient. This is supported by the study done by Won Jung Shin et al(16) and Hossam et al.(12)

Leak pressure (mean) in Group I was $26.23 \pm 1.993$, Group C was $20.58 \pm 1.615$ and in Group P was $27.75 \pm 2.295$ $\mathrm{cm}$ of water. This difference is statistically significant $(\mathrm{P}<$ 0.001) showing that Classic LMA forms a better seal in comparison to I-Gel or LMA Proseal. I-Gel and Proseal LMA are comparable in relation to the airway leak pressure.

Our study results are consistent with the study done by Won Jung Shin et al(16) who compared I-Gel, Proseal LMA and classic LMA in anaesthetised patients. The airway leak pressures of the I-Gel group $\left(27.1 \pm 6.4 \mathrm{~cm} \mathrm{H}_{2} \mathrm{O}\right)$ and pLMA group $\left(29.8 \pm 5.7 \mathrm{~cm} \mathrm{H} \mathrm{H}_{2} \mathrm{O}\right)$ were significantly higher than that of the cLMA group $\left(24.7 \pm 6.2 \mathrm{~cm} \mathrm{H}_{2} \mathrm{O}\right)$.

Airway sealing pressures are in accordance to the results of study done by I Singh et al,(17) who had compared the clinical performance of I-Gel ${ }^{\mathrm{TM}}$ with LMA-Proseal ${ }^{\mathrm{TM}}$ in Elective Surgeries. They found the airway sealing pressure was higher with LMA-ProSeal $\left(29.6 \mathrm{~cm} \mathrm{H}_{2} \mathrm{O}\right)$ than with I-Gel $(25.27 \mathrm{~cm}$ $\mathrm{H}_{2} \mathrm{O}$ ).

Although, this was statistically significant finding, but the airway sealing pressure of I-Gel and Proseal LMA are also within normal limit and effective in preventing aspiration.

Hossam et al(12) also had similar results where Leak pressure was significantly higher among patients of the I-Gel group $\left(25.62 \pm 4.9\right.$ versus $21.2 \pm 7.7 \mathrm{~cm} \mathrm{H}_{2} \mathrm{O}$ in LMA group; $\mathrm{P}<0.016$ ).

There was no case of post-operative sore throat in the Group I ( 0 \%), while 7 patients (17.5\%) in Group C (17.5\%) and 2 patients (5\%) in Group P developed sore throat. This difference among the three groups is statistically significant. These results are supported by Won Jung Shin et al.(16)

Christiaan Keijzer et al(18) in a randomised comparison of post-operative throat and neck complaints found the incidence of sore throat was significantly lower with the I-Gel than with La Premiere_ Disposable Laryngeal Mask at 1 (6 vs. 32), 24 (7 vs. 48) and $48 \mathrm{~h}$ (5 vs. 25).

In contrast, Hossam et al(12) did not find any significant difference between I-Gel and classic LMA in relation to the incidence of postop sore throat.

In our study, only one case of blood staining was seen in IGel group. There were only 3 cases in C Group and 5 cases in $P$ Group. The incidence of blood staining of device among the three (I, C, P) groups is not significant. We did not observe any other complication of coughing, gaging, vomiting, aspiration, regurgitation or bronchospasm in three groups.

Our study results accord with the study done by WonJung Shin et al,(16) who stated that the pLMA and cLMA were associated with increased rates of postoperative blood staining compared with the I-Gel, but the difference was not statistically significant. The inflatable cuffs of the pLMA and cLMA have the potential to cause complications such as mucosal injury, sore throat, airway obstruction and gastric insufflation(19) in contrast with the I-Gel without an inflatable cuff. However, the I-Gel could cause complications such as nerve injury, regurgitation and aspiration. $(20,21)$

\section{CONCLUSION}

I-Gel, the new supraglottic device is easier to insert in comparison to Proseal or Classic LMA with a high success rate of insertion in the first attempt and a very short time of insertion. The new non-inflatable supraglottic airway device has a slightly higher airway leak pressure compared to classic LMA. I-Gel has no significant incidence of post-operative sore throat or any complications. I-Gel is a better alternative among the available supraglottic devices.

\section{REFERENCES}

[1] Brain AIJ. The laryngeal mask: a new concept in airway management. British Journal of Anaesthesia 1983;55(8):801-5.

[2] Wilkins CJ, Cramp PG, Staples J, et al. Comparison of the anesthetic requirement for tolerance of laryngeal mask airway and endotracheal tube. Anesth Analg 1992;75(5):794-7.

[3] Joshi GP, Inagaki Y, White PF, et al. Use of the laryngeal mask airway as an alternative to the tracheal tube during ambulatory anesthesia. Anesth Analg 1997;85(3):573-7.

[4] Hohlrieder M, Brimacombe J, von Goedecke A, et al. Postoperative nausea, vomiting, airway morbidity and analgesic requirements are lower for the ProSeal laryngeal mask airway than the tracheal tube in females undergoing breast and gynaecological surgery. Br J Anaesth 2007;99(4):576-80.

[5] Bein B, Scholz J. Supraglottic airway devices. Best Pract Res Clin Anaesthesiol 2005;19(4):581-93.

[6] Brain AI. The development of the Laryngeal Mask-a brief history of the invention, early clinical studies and experimental work from which the Laryngeal Mask evolved. Eur J Anaesthesiol Suppl 1991;4:5-17.

[7] Brimacombe J, Keller C, Fullerkrug B, et al. A multicenter study comparing the ProSeal and Classic laryngeal mask airway in anesthetized, nonoparalyzed patients. Anesthesiology 2002;96(2):289-95.

[8] Asai T, Kawashima A, Hidaka I, et al. The laryngeal tube compared with the laryngeal mask: insertion, gas leak pressure and gastric insufflations. $\mathrm{Br} \mathrm{J}$ Anaesth 2002;89(5):729-32.

[9] Luba K, Cutter TW. Supraglottic airway devices in ambulatory settings. Anesthesiology Clin 2010;28(2):295-314.

[10] Brain Al, Verghese C, Strube PJ. The LMA 'ProSeal'-a laryngeal mask with an oesophageal vent. Br J Anaesth 2000;84(5):650-4.

[11] Richez B, Saltel L, Banchereau F, et al. A new single use supraglottic airway device with a non-inflatable cuff and an esophageal vent: an observational study of the I-gel. Anesth Analg 2008;106(4):1137-9.

[12] Helmy AM, Atef HM, El-Taher EM, et al. Comparative study between I-gel, a new supraglottic airway device, and classical laryngeal mask airway in anesthetized spontaneously ventilated patients. Saudi J Anaesth 2010;4(3):131-6. 
[13] Acott CJ. Extraglottic airway devices for use in diving medicine - part 3: the I-gel ${ }^{\mathrm{TM}}$. Diving Hyperbaric Med. 2008;38(3):124-7.

[14] Gatward JJ, Cook TM, Seller C, et al. Evaluation of the size 4 I-gel airway in one hundred paralysed patients. Anaesthesia 2008;63(10):1124-30.

[15] Gasteiger L, Brimacombe J, Perkofer D, et al. Comparison of guided insertion of the LMA Proseal TM vs the I-gel Anaesthesia 2010;65(9):913-6.

[16] Shin WJ, Cheong YS, Yang HS, et al. The supraglottic airway I-gel in comparison with ProSeal laryngeal mask airway and classic laryngeal mask airway in anaesthetized patients. Eur J Anaesthesiol 2010;27(7):598-601.

[17] Singh I, Gupta M, Tandon M, et al. Comparison of clinical performance of I-Gel ${ }^{\mathrm{TM}}$ with LMA-Proseal ${ }^{\mathrm{TM}}$ in elective surgeries. Indian J Anaesth 2009;53(3):302-5.
[18] Keijzer C, Buitelaar DR, Efthymiou KM, et al. A comparison of postoperative throat and neck complaints after the use of the I-gel and the la premiere disposable laryngeal mask: a double-blinded, randomized, controlled trial. Anesth Analg 2009;109(4):1092-5.

[19] Cook TM, Lee G, Nolan JP. The ProSeal laryngeal mask airway: a review of the literature. Can J Anaesth 2005;52(7):739-60.

[20] Gibbison B, Cook TM, Seller C. Case series: protection from aspiration and failure of protection from aspiration with the I-gel airway. $\mathrm{Br} \mathrm{J}$ Anaesth 2008;100(3):415-7.

[21] Theron AD, Loyden C. Nerve damage following the use of an I-gel supraglottic airway device. Anaesthesia 2008;63(4):441-2. 